\title{
Short Communication: Exploring Patterns of Variation in Conformation Data by Means of Principal Components Analysis
}

\author{
M. del P. Schneider and W. F. Fikse ${ }^{1}$ \\ Interbull Centre, Department of Animal Breeding and Genetics, Swedish University of Agricultural Sciences, PO Box 7023, SE-75007 \\ Uppsala, Sweden
}

\begin{abstract}
To explore patterns of variation in Holstein conformation data, 19 genetic correlation matrices from the May 2006 Interbull routine international sire genetic evaluation were reparameterized using an approximate factor analysis. For the udder traits teat length and rear teat placement, only 3 and 5 principal components explained 98 and $99 \%$ of the total variation, respectively, whereas 9 to 13 were necessary for overall conformation traits. The absolute deviations of the reparameterized genetic correlations from the initial genetic correlations were lower than 0.03 when $98 \%$ of the total variation was considered. The countries with the most frequent large contributions $(>0.30)$ to the second eigenvector for the body, mobility, and overall traits were Australia, New Zealand, Switzerland, Estonia, and Poland. For the udder traits no clear pattern was observed.
\end{abstract}

Key words: approximate factor analysis, conformation trait, genetic correlation, international sire evaluation

The estimation of the genetic correlations among countries is required for the international dairy sire genetic evaluations carried out by Interbull (International Bull Evaluation Service). The evaluations are conducted using multiple-trait across-country evaluations (MACE; Schaeffer, 1994), where similar traits (e.g., stature) measured in different countries are considered as different but correlated traits. The across-country genetic correlations are often difficult to estimate due to weak genetic links between dairy populations in different countries, as most of the bulls only have daughters in one country. The increasing number of participating countries and traits evaluated make the estimation even a more difficult task. Thus, the estimation of genetic parameters is one of the technical problems that Interbull faces today. Therefore, the application of methods that exploit patterns in genetic

Received December 17, 2007.

Accepted April 10, 2008.

${ }^{1}$ Corresponding author: Freddy.Fikse@hgen.slu.se correlation matrices that could result in a reduction of the number of parameters to be estimated is crucial.

Principal components (PC) and factor analysis are 2 potential approaches that can be used to identify patterns in the data and to summarize the data with little loss of information. Leclerc et al. (2005) has shown that a PC and an approximate factor analysis (AFA) gave good approximations of estimated genetic correlations for milk production in international sire genetic evaluations. The objective of this study was to explore, using an approximate factor analysis approach, patterns of variation in the genetic correlation matrix of Holstein conformation traits.

Genetic correlations (rG) of 19 Holstein conformation traits from the May 2006 Interbull routine sire genetic evaluation were used (Interbull, 2006). The rG were estimated in the March 2006 test evaluation using deregressed national breeding values and their effective daughter contribution, and an expectation maximization (EM)-REML (Klei and Weigel, 1998) procedure. In total there were 20 countries, but not all countries were included for the various traits. Type traits were grouped in body, mobility, udder traits, and overall traits.

The rG matrix of each conformation trait was reparameterized using an AFA. First, PC for the rG matrix were obtained by means of a canonical decomposition of the $\mathrm{rG}$ matrix: $r G=U \cdot D \cdot U^{\prime}=V \cdot V^{\prime}$, with $U \cdot U^{\prime}=I_{M}$ and $U^{\prime} \cdot U=I_{N}$, where $D$ is the diagonal matrix of eigenvalues, $V$ is the corresponding set of orthogonal eigenvectors, $V$ is $U \cdot D^{1 / 2}$, and $I_{i}$ is the identity matrix of dimension $i$. The eigenvectors of the genetic (co)variance matrix define the so-called PC, which are independent linear functions of the variables considered. Their statistical significance is assessed through the amplitude of the corresponding eigenvalues.

The rank of the rG matrix can be reduced by setting to zero the smallest eigenvalues in the $D$ matrix and deleting the corresponding eigenvectors from $U: P^{*}=U^{*} \cdot D^{*} \cdot U^{* \prime}=V^{*} \cdot V^{* \prime}$, with $U^{*} \cdot U^{* \prime}=I_{N}$ and $U^{*} \cdot U^{*}=I_{M}$, where $P^{*}$ is a matrix with rank 
Table 1. Number of principal components necessary to retain 98 and $99 \%$ of the total variation, and cumulative proportions of the variances, for 19 Holstein conformation traits

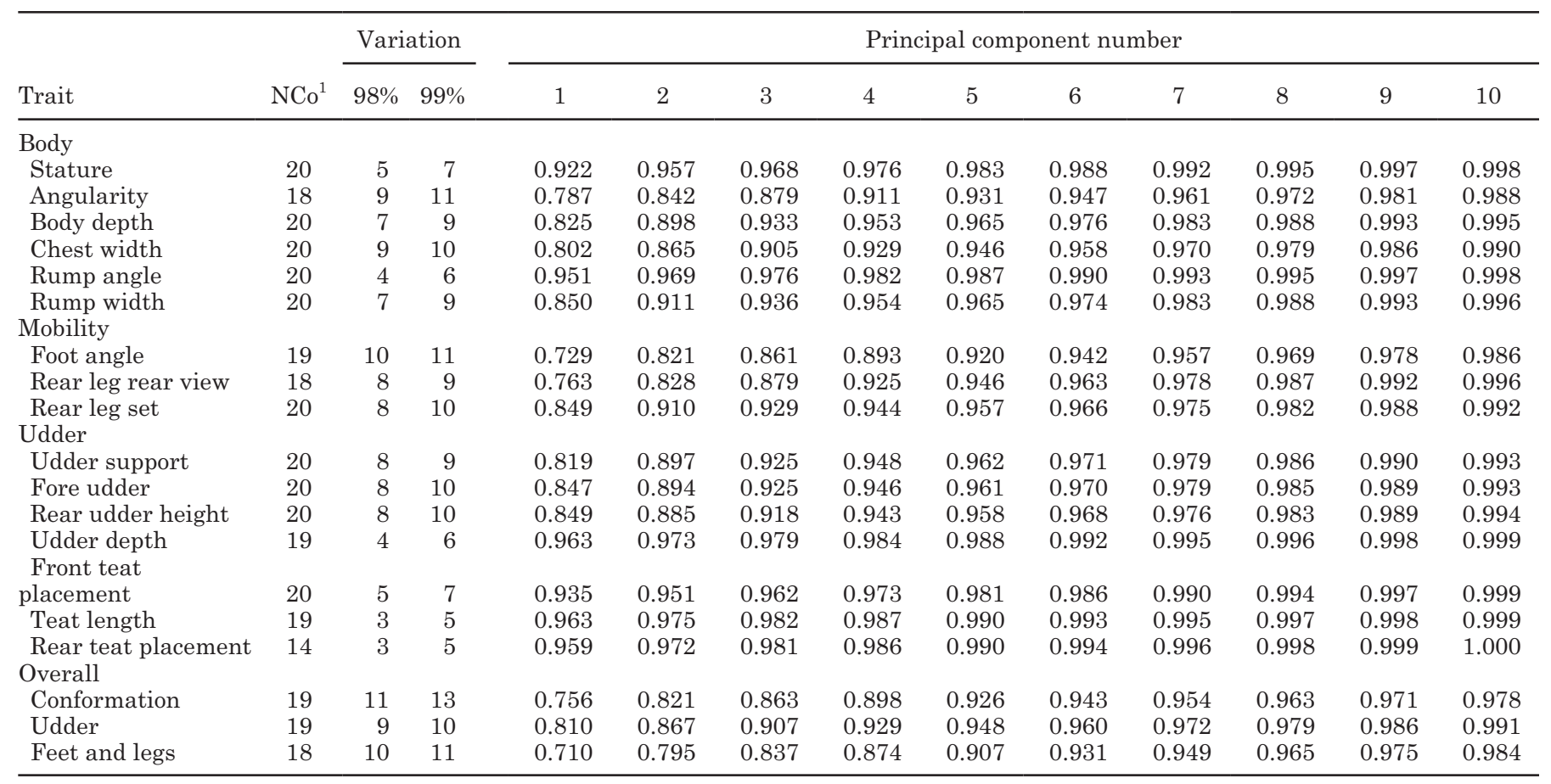

${ }^{1} \mathrm{NCo}=$ number of participating countries in the May 2006 international genetic evaluation. Countries not included in the evaluation: Angularity: Switzerland, Red Holstein (CHR) and New Zealand (NZL); Foot angle: NZL; Rear leg rear view: Czech Rep. (CZE) and NZL; Udder depth: NZL; Teat length: NZL; Rear teat placement: CHR, CZE, Hungary (HUN), Japan (JPN), Poland (POL) and Rep. of South Africa (ZAF); Overall conformation: ZAF; Overall udder: ZAF; Overall feet and legs: NZL and ZAF.

$N<M, \quad D^{*}$ is the matrix of modified eigenvalues, $U^{*}$ contains the corresponding set of orthogonal eigenvectors, and $V^{*}$ is $U^{*} \cdot D^{* 1 / 2}$.

The $P^{*}$ matrix does not necessarily have 1 on the diagonal anymore, and to obtain the reparameterized genetic correlation matrix $\left(\mathbf{r G}^{*}\right)$, matrix $P^{*}$ can be rescaled $\left[r G^{*}{ }_{i j}=P^{*}{ }_{i j} / \sqrt{P^{*}{ }_{i i} \times P^{*}}{ }_{j j}\right.$, referred to as the PC approach by Leclerc et al. (2005)], or a diagonal matrix $F$ can be added to make the diagonal elements of the $\mathrm{rG}^{*}$ matrix equal to 1 . The elements of $F$ can be considered the specific factors in the factor analysis. Here, these elements were defined as constraints [ $\left.F=I-\operatorname{diag}\left(P^{*}\right)\right]$, and are not estimated at the same time as $P^{*}$, as is done in the formal factor analysis. With the AFA approach, there is a reduction of the numberofparameterstobeestimated: $N(2 M-N+1) / 2$ (Meyer and Kirkpatrick, 2005). However, the $\mathrm{rG}^{*}$ matrix remains full rank.

For the udder traits teat length and rear teat placement, only 3 and 5 PC were enough to explain 98 and $99 \%$ of the total variation, respectively, whereas 8 to 10 were needed for fore udder and rear udder height (Table 1). Differences in the number of PC were also found among body traits, the extreme ones being rump angle (4 to $6 \mathrm{PC}$ ) and angularity (9 to $11 \mathrm{PC}$ ). For the linearly scored feet and legs and overall composite traits (overall conformation, overall udder, and overall feet and legs) 8 to 11 and 9 to 13 PC were needed to explain 98 and $99 \%$ of the variation, respectively. The greater number of PC needed for the mobility and overall traits may reflect variability in trait definition. Udder support needing equally many PC to retain $98 \%$ of the variation as 2 of the feet and legs traits was somewhat surprising because more "harmonization" in trait definition was expected.

A large difference in the number of $\mathrm{PC}$ needed to retain a certain percentage of variation was found among the traits. This variation could reflect differences in trait definition, models, and genotype by environment interactions (e.g., tied vs. free-stalls for feet and legs traits).

A common question in $\mathrm{PC}$ analysis is how many $\mathrm{PC}$ are necessary to retain, and it should result in a balance between the reduction of number of parameters and loss of information. The percentage of the variation chosen (i.e., 98\%) was somewhat arbitrary, and it is one of the approaches suggested in the literature. 
Table 2. Maximum, minimum, average, and average absolute (Abs) deviations of the reparameterized genetic correlations with an approximate factor approach from the genetic correlations $(\mathrm{rG})$, for the principal component that retained $98 \%$ of the total variation for 19 Holstein conformation traits

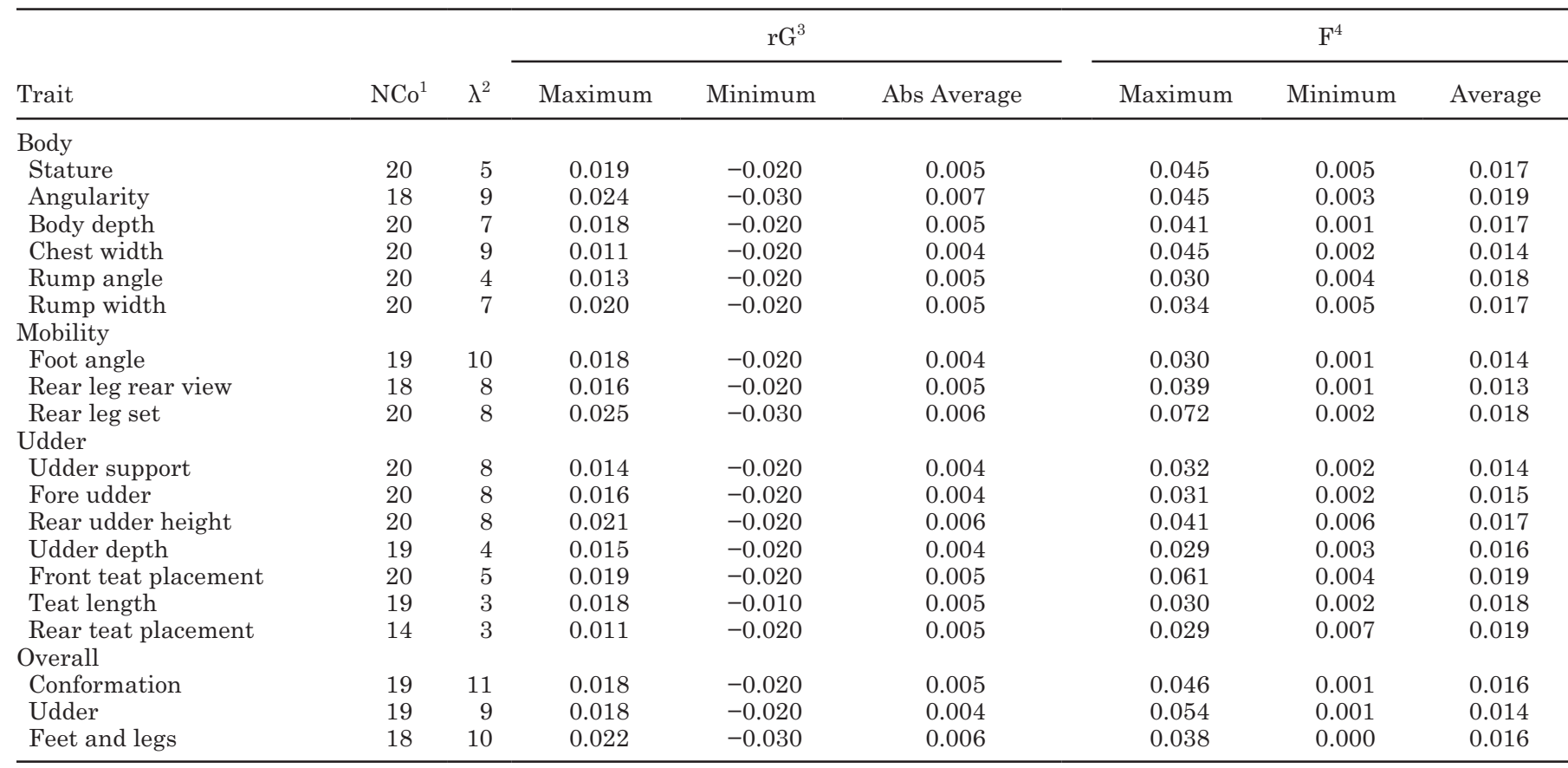

${ }^{1} \mathrm{NCo}=$ number of participating countries in the May 2006 international genetic evaluation.

${ }^{2}$ Number of eigenvalue and corresponding eigenvector used to reparameterize the genetic correlation matrix.

${ }^{3}$ The average deviation for the genetic correlations was -0.001 for all conformation traits.

${ }^{4}$ Elements in diagonal matrix $\mathrm{F}\left[F=\mathrm{I}-\operatorname{diag}\left(\mathrm{P}^{*}\right)\right]$.

However, if we want to retain $99 \%$ of the variation instead (Table 1), it can be achieved by only keeping 1 or 2 more PC.

For $r G$ (off-diagonal elements of the matrix), the maximum and minimum deviations were $<0.03$ in all cases, and the absolute deviations ranged from 0.004 to 0.007 when at least $98 \%$ of the variation was retained (Table 2). Therefore, genetic correlations obtained with the reparameterized $\mathrm{rG}$ matrix were very close to the "initial" $r G$. The average values of $\mathrm{F}$ (elements added to the diagonal) were $<0.020$. These F-values represent the variation of the factors specific for each country.

For teat length, the trait with the highest leading eigenvalue, only $1 \mathrm{PC}$ was needed to obtain an average absolute deviation $<0.03$, whereas $4 \mathrm{PC}$ were needed for overall conformation (Table 3). For the elements of F, 2 and $9 \mathrm{PC}$ were necessary to get an average deviation $<0.03$ for teat length and overall conformation, respectively.

For body traits, the countries with the most frequent large contributions to the second eigenvector $(>0.30)$ were Australia, New Zealand, Estonia, Poland, and Japan, and for mobility traits, Switzerland and Australia (Table 4). Australia, Poland, and the Nordic countries (DFS; joint evaluation from Denmark, Fin- land, and Sweden) were the countries with the most frequent large contribution for overall traits. These groups of countries were relatively different from the rest. Australia and New Zealand have different production systems compared with northern hemisphere countries. For Estonia, the large contributions may be related to high standard error of the estimated genetic correlations, because of the relatively weak genetic links (i.e., few commons bulls with other countries). No clear pattern was observed for the udder traits, perhaps indicating more agreement in trait definition across countries.

This type of analyses could provide useful information to breeders associations (e.g., the World HolsteinFriesian Federation) in their efforts to harmonize type classification across countries: it tells which traits are similarly defined across countries and which countries have deviating trait definitions.

Results showed that by keeping several PC that explained $98 \%$ of the total variation, absolute deviations $<0.03$ were obtained. Retaining $98 \%$ of the variation by considering $3 \mathrm{PC}$ for teat length would reduce the number of parameters to be estimated from 190 to 54 for the approximate factor analysis. The reduction for the "worst" trait, overall conformation, would be from 
Table 3. Maximum, minimum, average, and average absolute (Abs) deviations of the reparameterized genetic correlations with an approximate factor approach from the genetic correlations $(\mathrm{rG})$, for teat length (TL) and overall conformation (OC)

\begin{tabular}{|c|c|c|c|c|c|c|c|c|c|c|c|c|c|c|c|c|}
\hline \multirow{2}{*}{$\lambda^{1}$} & & & \multicolumn{8}{|c|}{$\mathrm{rG}$} & \multicolumn{6}{|c|}{$\mathrm{F}^{3}$} \\
\hline & \multicolumn{2}{|c|}{ Cum. prop. ${ }^{2}$} & \multicolumn{2}{|c|}{ Maximum } & \multicolumn{2}{|c|}{ Minimum } & \multicolumn{2}{|c|}{ Average } & \multicolumn{2}{|c|}{ Abs Average } & \multicolumn{2}{|c|}{ Maximum } & \multicolumn{2}{|c|}{ Minimum } & \multicolumn{2}{|c|}{ Average } \\
\hline 1 & 0.963 & 0.756 & 0.034 & 0.311 & -0.052 & -0.290 & -0.002 & -0.013 & 0.009 & 0.049 & 0.100 & 0.600 & 0.010 & 0.126 & 0.037 & 0.244 \\
\hline 2 & 0.975 & 0.821 & 0.025 & 0.155 & -0.023 & -0.153 & -0.001 & -0.010 & 0.006 & 0.041 & 0.068 & 0.328 & 0.010 & 0.116 & 0.025 & 0.179 \\
\hline 3 & 0.982 & 0.863 & 0.018 & 0.140 & -0.015 & -0.113 & -0.001 & -0.007 & 0.005 & 0.031 & 0.030 & 0.301 & 0.002 & 0.040 & 0.018 & 0.137 \\
\hline 6 & 0.993 & 0.943 & 0.006 & 0.028 & -0.007 & -0.060 & 0.000 & -0.003 & 0.002 & 0.012 & 0.014 & 0.091 & 0.001 & 0.015 & 0.007 & 0.057 \\
\hline 7 & 0.995 & 0.954 & 0.005 & 0.025 & -0.005 & -0.034 & 0.000 & -0.003 & 0.001 & 0.010 & 0.012 & 0.083 & 0.001 & 0.014 & 0.005 & 0.046 \\
\hline 8 & 0.997 & 0.963 & 0.003 & 0.022 & -0.005 & -0.034 & 0.000 & -0.002 & 0.001 & 0.008 & 0.011 & 0.082 & 0.001 & 0.002 & 0.003 & 0.037 \\
\hline 9 & 0.998 & 0.971 & 0.003 & 0.022 & -0.004 & -0.036 & 0.000 & -0.002 & 0.001 & 0.007 & 0.010 & 0.061 & 0.001 & 0.001 & 0.002 & 0.029 \\
\hline 10 & 0.999 & 0.978 & 0.002 & 0.018 & -0.003 & -0.026 & 0.000 & -0.001 & 0.001 & 0.006 & 0.004 & 0.048 & 0.000 & 0.001 & 0.001 & 0.022 \\
\hline 11 & 0.999 & 0.984 & 0.001 & 0.018 & -0.002 & -0.023 & 0.000 & -0.001 & 0.000 & 0.005 & 0.004 & 0.046 & 0.000 & 0.001 & 0.001 & 0.016 \\
\hline
\end{tabular}

${ }^{1}$ Number of eigenvalue and corresponding eigenvector used to reparameterize the genetic correlation matrix.

${ }^{2}$ Cum. Prop. $=$ cumulative proportion explained variance by the eigenvalues/eigenvectors.

${ }^{3}$ Elements in diagonal matrix $\mathrm{F}\left[F=\mathrm{I}-\operatorname{diag}\left(\mathrm{P}^{*}\right)\right]$.

190 to 154 . Considering all 19 traits, only 2,138 parameters would have to be estimated instead of the 3,668 of the unstructured genetic correlation matrices assumed today.

The identification of countries that contribute to a large variation could be used to select groups of coun-

Table 4. Number of times a country had a large contributions $(>0.30)$ to the second eigenvector for body, mobility, udder, and overall traits

\begin{tabular}{|c|c|c|c|c|}
\hline Country $^{1}$ & $\operatorname{Body}(6)^{2}$ & Mobility (3) & Udder (7) & Overall (3) \\
\hline AUS & 5 & 2 & 3 & 3 \\
\hline BEL & - & - & 2 & - \\
\hline CAN & - & - & 1 & - \\
\hline CHE & 2 & 2 & 1 & - \\
\hline CHR & - & 2 & 2 & 1 \\
\hline CZE & 1 & - & - & - \\
\hline DEU & - & - & - & - \\
\hline DFS & - & - & - & 3 \\
\hline ESP & - & - & - & - \\
\hline EST & 3 & - & 1 & 1 \\
\hline FRA & 2 & - & 2 & - \\
\hline GBR & - & 1 & 1 & 1 \\
\hline HUN & 1 & - & 2 & - \\
\hline ITA & - & - & 3 & - \\
\hline JPN & 3 & - & - & - \\
\hline NLD & - & - & - & - \\
\hline NZL & 4 & 1 & 1 & 1 \\
\hline POL & 3 & - & 2 & 3 \\
\hline USA & - & - & 2 & - \\
\hline $\mathrm{ZAF}$ & - & 1 & - & - \\
\hline
\end{tabular}

${ }^{1}$ Country codes: AUS $=$ Australia, BEL $=($ Walloon part of $)$ Belgium, CAN = Canada, CHE $=$ Switzerland, CHR $=$ Switzerland $($ Red Holstein), CZE = Czech Rep., DEU = Germany, DFS = Denmark + Finland + Sweden, ESP $=$ Spain, EST $=$ Estonia, FRA = France, GBR = United Kingdom, HUN = Hungary, ITA = Italy, JPN = Japan, NLD $=$ Netherlands $(+$ Flemish part of Belgium) NZL $=$ New Zealand, POL $=$ Poland, USA $=$ United States, ZAF $=$ Rep. of South Africa.

${ }^{2}$ Number of traits evaluated in each groups shown in parentheses.s tries to be used in the estimation of genetic correlations in the 2-step approach used by Leclerc et al. (2005) for milk yield, with the final aim to reduce the number of parameters to be estimated without losing too much information. In the Leclerc et al. (2005) study, Australia and New Zealand had the largest contribution to the second eigenvector, and Poland, Czech Republic, and New Zealand to the third eigenvector. These countries had often large contributions for conformation traits in this study as well. However, it is difficult to identify a specific reason why some of these countries are different from the rest, but since it is observed for more than one trait group (i.e., production and conformation) it may be interpreted as evidence for genotype by environment interaction.

The large variability found among the type traits will require different selection according to the trait under study. Approximate factor analysis is an approximate method to estimate genetic correlations; more research is still needed in the field.

\section{REFERENCES}

Interbull. 2006. Interbull Routine Genetic Evaluation for Conformation Traits February 2006. http://www-interbull.slu.se/ conform/framesida-conf.htm. Accessed Feb. 15, 2006.

Klei, B., and K. A. Weigel. 1998. A method to estimate correlations among traits in different countries using data on all bulls. Interbull Bull. 17:8-14.

Leclerc, H., W. F. Fikse, and V. Ducrocq. 2005. Principal components and factorial approaches for estimating genetic correlations in international sire evaluation. J. Dairy Sci. 88:3306-3315.

Meyer, K., and M. Kirkpatrick. 2005. Restricted maximum likelihood estimation of genetic principal components and smoothed covariance matrices. Genet. Sel. Evol. 37:1-30.

Schaeffer, L. R. 1994. Multiple-country comparison of dairy sires. J. Dairy Sci. 77:2671-2678. 